\title{
First-Principles Calculations: The Elemental Transition Metals and Their Compounds
}

\author{
R. E. Watson, G. W. Fernando, M. Weinert, and J. W. Davenport
}

Department of Physics

Brookhaven National Laboratory

Upton, New York 11973

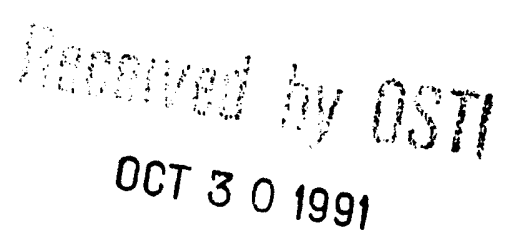

\begin{abstract}
If done with sufficient care, present day a priori theory yields calculated enthalpies of formation whose agreement with experiment (when such data is available) is of the order of the experimental scatter. Comparisons will be made for the Pt-Ti systems for which such data exist and for which one crystal structure involves atomics sites of low symmetry. Two other cases will be considered for which there is no direct experimental heats data. The first of these will be the structural stabilities of the $4 d$ elemental metals. Such structural stabilities have been an issue of contention between electronic structure theorists and those who construct phase diagrams for some twenty-five years. The second involves the energetics of forming metal adlayers and artificial multilayers. The distortion energies associated with the requirement that adlayers (or multilayers) conform to some given substrate are often the controlling factors in the fabrication of multilayer materials. This contribution is best understood by invoking a combination of elemental structural promotion energies plus elastic distortions from these structures. As will be seen, the fabrication of multilayers also involves a term not normally encountered in bulk phase diagram considerations, namely the difference in surface energies of the two multilayer constituents.
\end{abstract}

The submitted manuscript has been authored under contract DE-AC02-76CH00016 with the Division of Materials Sciences, U.S. Department of Energy. Accordingly, the U.S. Government retains a nonexclusive, royalty-free license to publish or reproduce the published form of this contribution, or allow others to do so, for U.S. Government purposes. 


\section{Introduction}

It has become quite commonplace for electronic structure calculations, of varying rigor, to yield the total energy of a solid. This offers the prospect of estimating the stabilities of phases which are either unavailable or inaccessible experimentally and this, in turn, has implications for making phase diagram predictions. This paper will concentrate on predictions for transition metals and their ordered compounds which employ full potential, augmented basis set calculations. An augmented basis set scheme employs a basis set of plane waves, Gaussians, Slater-type orbitals or the like in the interstitial regions of the crystal and augments these by explicit solutions of radial wave equations in atomic spheres at each atomic site. In our view, such augmented schemes offer the greatest present-day rigor for the treatment of transition metal systems. A full potential treatment implies carrying nonspherical as well as spherical terms in the crystal charge density and in the crystal potential. These nonspherical terms will, for example, contribute to the mixing of one-electron orbitals of differing $\ell$ within the atomic spheres. (The choice of the division of the crystal space into interstitial and atomic sphere regions becomes less sensitive when full potentials are employed.) In what follows, we will first consider the relative stabilities of the elemental transition metals on going from one crystal structure to another. This issue represents the first quantitative confrontation [67 Rud] between the condensed matter theorists and the CALPHAD (CALculation of PHAse Diagram) practitioners [70 Kau; 88 Sau; $88 \mathrm{Gui}$ ]. This will be followed by some considerations of the energetics of multilayer materials and systems with metal adlayers - the latter is relevent to the former since multilaye e fabricated by adlaying one material upon another. We will then consider predictions for the Pt-Ti system. This system was chosen because of the availability [90a Sel; $90 \mathrm{~b} \mathrm{Sel} ; 85 \mathrm{Col} ; 88 \mathrm{Top}]$ of experimental heats of formation data for a number of the observed phases, including ones involving "ill-packed" as well as close-packed crystal structures. (We expect that Gauchon will discuss the experimental situation with this system, elsewhere in this symposium). 
The calculations which will be referred to in this article are large scale, computationally expensive efforts. In the paper which follows, Zunger describes a less expensive and less rigorous scheme which allows him and his coworkers to estimate the relative stabilities of large numbers of possible competing phases. The two approaches are complimentary to one another, as are other schemes which are more approximate than Zunger's.

An estimate of phase stability involves the comparison of the total energy of some given phase with the energy of another system. For example, the stability of an elemental $b c c$ phase relative to its $f c c$ counterpart is

$$
E_{b c c}-E_{f c c}
$$

and the heat of formation of some binary compound is

$$
\Delta H=E_{c}-\sum_{i} x_{i} E_{i}
$$

where $E_{c}$ is the calculated total energy of the compound, $E_{\boldsymbol{i}}$ is the calculated total energy of element $i$ (usually in its solid form) and $x_{i}$ is the concentration of element $i$ in the compound. The cohesive energy of an elemental solid is

$$
H_{c o h}=E_{s o l}-E_{a t}
$$

where $E_{a t}$ is the energy of the free elemental atom. One can expect to do well when evaiuating the above expressions when the equivalent computational scheme does similarly well for the various energies represented in the expression. As a rule, computations do worst for $H_{\text {coh }}$ where the comparison is between a solid and a free atom. Calculations tend to overestimate $H_{\text {coh }}$ by a significant amount for open shell systems, i.e. theory is doing worse for the open shell free atom than it is for the solid. Done with sufficient care, current theory is in reasonable accord with experiment when estimating the heats of formation of compounds. 


\section{The Lattice Stabilities of the Elemental Transition Metals}

One of the first encounters between the CALPHAD workers who calculate phase diagrams and the electronic structure theorists occurred [67 Rud] at the Battelle metallurgy meeting in Switzerland in the mid 60's. In the treatment of a terminal solution phase, say of $b c c \mathrm{Nb}$ dissolved at a dilute concentration in $f c c P d$, the CALPHAD practice would be to take the energy necessary to promote elemental $N b$ from the $b c c$ to the $f c c$ structure and this would be taken as the lead energy term in the energy of the terminal phase. Although modern day total energy estimates were not then available for such promotion energies, Mott and Friedel observed [ $67 \mathrm{Rud}$ ] that such $f c c-b c c$ promotion energies would be roughly an order of magnitude larger (though agreeing in sign) than the values then finding favor with CALPHAD practitioners [70 Kau] - values which continue to find favor with many workers to this day. Pettifor's pioneering tight - binding estimates [77 Pet] of some years later lent evidence in support of Mott's and Friedel's views. The situation is even more extreme for the $f c c-h c p$ energy differences, which are smaller due to the simularity in the packing of the two struciures, but for which there is disagreement as to the sign of the energy difference for those metals which have the bcc structure in their ground state. Consider, for example, the $M o-R h$ system, for which at intermediate composition there is a substitutional $h c p$ phase at just the electron to atom ratios for which the $h c p$ structure might be expected to occur for an elemental transition metal system. In the CALPHAD construct, $b c c M o$ and $f c c R h$ are promoted to $h c p$ structures; the construct is in some numerical difficulty if $h c p M o$ is not found to be stabler than $f c c M o$. Total energy calculations have consistently yielded [ $85 \mathrm{Skr} ; 90 \mathrm{Fer} ; 85 \mathrm{Dav}$ ] the contrary for the bcc metals of the $V$ and $C r$ columns of the periodic table, i.e. all estimates indicate the $f c c$ structure stabler than the hcp. The problem, in our view, is that the standard phase diagram construct does not sufficiently admit the importance of band filling, i.e. electron to atom ratio, in such structures when formed at intermediate composition upon alloying.

Here we will consider the $f c c-b c c$ energy differences for the $4 d$ transition metal row 
which complement earlier published results $[90 \mathrm{Fer}]$ for the $5 d$ row. In both cases, these are full potential linear augmented Slater-type orbitals (LASTO) calculations employing Heden-Lundqvist type local potentials [71 Hed]. The present calculations are fully relativistic, unlike the previous results for the $5 d$ which omitted spin-orbit effects in the valence bands. The results appear in Fig. 1. These are compared with the estimates of Saunders, Miodownik, and Dinsdale who asked how far the original values of Kaufman [70 Kau] could be pushed towards the band theory total energy estimates while remaining plausible within che CALPHAD constructs. Saunders et al.'s values agree in sign but are much larger in magnitude than Kaufman's and except for $R u$ and $R h$ the present total energy results are in semi-quantitative agreement with these values. The same situation holds $[90 \mathrm{Fer}]$ in the $5 d$ row where there is semi-quantitative agreement with Sauders et al. except for $O s$ and Ir which are the $5 d$ counterparts of $R u$ and $R h$. It is expected that even more careful total energy estimates of these structural energy differences, if employing the same type of local density potential, will yield results which touch the plotted points on the figure. (The introduction of valence electron spin-orbit coupling has essentially no effect on these results.)

The experimentally observed elemental lattice volumes were used when calculating the total energies for the $f c c$ and $b c c$ structures of Fig. 1. As a rule, electronic structure calculations employing local density potentials have their mimima in total energies at lattice volumes whicii are somewhat smaller than those observed. The consequences of this for $h c p R u$ and for $f c c R h, P d$, and $A g$ are to be seen in Fig. 2. All show a lattice contraction associated with going to the energy minimum, which is of the order of one percent in lattice constant (hence three percent in volume) for $R u, R h$, and $P d$ and twice this for $A g$. The effect of going to the energy minimum is less than $0.01 \mathrm{eV} /$ atom in the total energies for $R u, F h$, and $P d$ and slightly greater than $0.01 \mathrm{eV} /$ atom for $A g$. Shifts of $0.01 \mathrm{eV} /$ atom are of no consequence to the results of Fig. 1 (though, of course, it is the difference in a pair of such shifts which actually enters a structural energy difference). It is 
computationally quite convenient to trace out the energy minumum, if desired, for most any elemental solid, but it becomes costly in the computer time for compounds (or elemental systems such as $\alpha M n$ ) having large numbers of atoms in the unit cell. Experience, such as that displayed in Fig. 2 suggests that under most circumstances, obtaining total energies at the observed lattice volumes is a satisfactory procedure.

\section{Adlayers and Multilayer Materials}

Artificial multilayered materials have been the object of considerable scientific and technological interest. Understanding them involves discerning what happens when an adlayer of one material is laid lown on a substrate of some different material. Of course, such adlayers are themselves of chemical and physical interest. Several factors contribute to the energetics of multilayer systems. Consider a crystalline substrate of element $B$, covered with a reasonably thick epitaxial layer of element $X$. Using the elemental solids $X$ and $B$ as reference materials, the heat of adsorption of such a layer is

$$
\Delta E_{A}=\gamma_{X}-\gamma_{B}+\zeta+\Delta E_{s t r}
$$

where $\gamma_{X}-\gamma_{B}$ is the difference in surface energy between having a surface of element $X$, rather than $B$. (In order that the solids exist, the $\gamma$ 's must be positive and, since two surfaces are created upon cleavage, the cleavage energy is $2 \gamma$. ) $\Delta E_{s t r}$ is the structural energy, if any, associated with preparing $X$ so that it lies in registry with $B$ and $\zeta$ is the energy associated with bonding at the interface. The number of bonds across the interface versus the number of bonds of an interface $X$ (or $B$ ) atom within an $X$ (or a $B$ ) layer depends on the packing of the layers but the ratio of these numbers suggests packing characteristic of 2:1 or 3:1 compounds. Calculated $\zeta$ are consistent [89 Wei] with calculated heats of formation for such compounds (providing one remembers that the $\Delta H$, of Eq. (2), is defined per atom, while $\zeta$ is defined per site on the surface, i.e. per pair of $X$ and $B$ atoms of the surface). Eq. (4) is clearly an over simplication; for example $\gamma_{X}, \zeta$, and $\Delta E_{s t r}$ may all vary with the thickness of adlayer $X$. Despite this, these quantities may be 
extracted from total energies obtained for bulk and slab samples and, once obtained, are found to be transferrable, thus providing an understanding of a variety of experimental trends.

In the case of a multilayer system, there are no free surfaces and Eq. (4) becomes

$$
\Delta E=2 \zeta+\Delta E_{\text {str }}
$$

Here $\zeta$ is multiplied by 2 since a unit cell of a multilayer system will have two interfaces, $B \rightarrow X \rightarrow B . \Delta E_{s t r}$ may have contributions from both the $X$ and $B$ layers depending on how the multilayer forms.

The distortion energy, $\Delta E_{s t r}$, is important to Eqs. (4) and (5) and examples of it are to be found in Fig. 3 where the cost of laying $R u, R h, P d$, and $A g$ down in register with the packing of $N b$ and $M o(110)$ planes. The energies are plotted as a function of the distance between planes, absissa's of 1 correspond to having the lattice volumes and undistorted $b c c$ structure of the $M o$ and $N b$ templates. The vertical arrows correspond to interplanar spacings yielding the atomic volumes associated with the energy minima of Fig. 2 (here it is appropriate to compare the lattice distortions associated with energy minima with the elemental volumes of Fig. 2 which are based on the same type of computation). The plotted results are based on LASTO calculations involving full potentials and spin-orbit coupling in the valence bands. In all cases the interplanar spacing relaxes so that the atomic volumes moves back towards, but never gets to, the volume of the element in its stable phase. The distortion energies for $A g$ are quite modest while those for $R u$ and $R h$ are substantial, thus $\Delta E_{s t r}$ is expected to strongly discourage laying $R u$ and $R h$ down epitaxially on such substrates while not being the controling factor in the case of $A g$. The horizontal lines in the middle of the figure are the structural promotion energies associated with taking these $f c c$ metals (in the case of $R u$, the $h c p$ metal) to the bcc structure at the same volume. It would appear that, in the case of the $M o(110)$ template, the distortion energies can be rather well understood in terms of $f c c \rightarrow b c c$ (or $h c p \rightarrow b c c$ ) structural 
promotion energies. However, in the case of $N b$, its size mismatch with $R u, R h, P d$, and $A g$ is more substantial and the resulting distortion energies reflect this.

The third energy factor, which is important to adlayer and, in turn, multilayer tendencies but which does not normally enter consideration of bulk alloy phase behavior, is the surface energy term of Eq. (4). These can be estimated by doing careful total energy calculations for slabs of irying thickness. Results for different surfaces of $b c c N b$ and $f c c$ $A g$ and $P d$ appear in '. $\quad:$ I. These were obtained [89 Wei] with full-potential linearized augmented plance wav iculations (FLAPW) for slabs which were unreconstructed and had spacings between acijacent atomic layers which equaled those of the bulk. The only direct experimental comparison which can be made is that for liquid metals; experimentally deduced $\gamma$ for the solid metals requires numerical manipulations which introduce uncertainties which are of the order of those associated with a priori estimates. Consider, first, the $\gamma$ reported as an energy per surface atom. The results show the closer-packed surfaces to have the smaller $\gamma$, i.e. the smaller energy cost associated with creating the surface. This follows because fewer bonds are cut, i.e. an atom keeps more of its bulk nearest neighbors, on the closer-packed surface. The results for $\gamma$ show less variation between faces, on the order of $10 \%$, when $\gamma$ is considered per unit area. This is the appropriate measure of surface energy when considering equilibrium crystal shapes and the like. If there were no face dependence in $\gamma$, as one has for a jellium metal, the equilibrium crystal shape would be a sphere because this would minimize the surface area and hence the total surface energy. Since the majority of elements do grow rather spherically, we would expect only small variations in the energy per unit area as is the case in Table I.

When comparing surface energies to bulk properties such as heats of formation, the energy per atom is the more useful measure. The calculation can be extended [ $89 \mathrm{Wei}$ ] to having monolayers of, say, $P d$ or $A g$ on, say, $N b$ and, using Eq. (4) a surface energy can be deduced for the monolayer. If for example, the resulting surface energy is greater than the elemental values of Table I, this would imply that, from surface energy considerations 
(there, of course, are also $E_{\text {str }}$ factors), it would be energetically favorable to add more $P d$ or $A g$ layers upon the first.

Inspecting Table I shows the $\gamma$ (per atom) to vary significantly from face to face. This suggests that the interplay of $\gamma$ with the other terms of Eq. (4) will cause the adsorption properties of some element to vary with different faces of the substrate. This is the cast. Also, it is seen that the surface energies of $N b$ are substantially larger than those for $A g$. Total energy calculations [89 Wei] for a monolayer of $A g$ adsorbed on $N b$ show it is energetically cheaper for $A g$ to cover the surface than it is to have s balling of the $A g$ so as to minimize $A g-N b$ contact. At first glance, this is surprising because the bulk phase diagram for $A g-N b$ shows them to be immiscible and to form no compound, i.e. $\zeta$ is positive, that is non-bonding. The lower surface energy of $A g$ has overpowered this nonbonding (and $\Delta E_{s t r}$ ). This situation has been observed experimentally: $A g$ does cover $N b$.

The situation is different for $N b-P d$. When $P d$ is laid down on $N b$, both the surface energies and the $\zeta$ overpower the $\Delta E_{s t r}$ causing $P d$ to wet the $N b$ substrate. However $\Delta E_{\text {str }}$ is larger, than it is for $A g$, and thick $P d$ layers, with each plane in register with the substrate, are not expected and do not occur. The surface energy term, of course, acts against $N b$ being laid down on $P d$. While neither we, nor to our knowledge anyone else, has estimates of $\gamma$ for ordered or disordered $P d-N b$ alloys, it appears energetically plausible that $N b$ once put down on the surface prefers to form compounds with $P d$, thus gaining the energy of formation while minimizing $\Delta E_{s t r}$ and surface terms. This appears to be the case experimentally. In low energy ion scattering studies [89 Jia] of $T a$ (the $5 d$ counterpart of $N b$ ) laid down $P d$, there are indications of $P d$ in the surface even after eight layers of $T a$ have been laid down, while complementary studies of $P d$ on $T a$ show the $T a$ to disappear after monolayer $P d$ coverage. There are also $x$-ray diffraction results for $N b$ - $P d$ multilayers which are understandable if the interfaces where $P d$ was laid down on $\mathrm{Nb}$ are compositionally sharp, while those where $N b$ went down on $P d$, have regions of 
substantial $P d-N b$ compositional mixing [91 Str].

In this section we have considered the consequences of Eqs (4) and (5) for adlayer and multilayer behavior. Total energy calculations can be used to estimate the terms entering these equations and these quantities are transferable. Several illustrations have been given suggesting how experimental trends can be anticipated or rationalized given these equations and the quantities entering them. In considering the formation of the multilayers, we have encountered a factor not normally invoked in bulk phase behavior, namely the surface energy term. In closing we might note that these surface energy factors are expected to change measurably when one goes from fabrication employing vacuum deposition techniques to those utilizing electrochemical deposition.

\section{The Pt-Ti Systems}

Experimental $\Delta H$ are available for most of the observed ordered phases in the $P t$ $T i$ system. There are calorametric results for $P t_{3} T i, P t T i$, and $P t T i_{3}$ as well as emf measurements for $\mathrm{Pt}_{8} \mathrm{Ti}$ and $\mathrm{Pt}_{3} \mathrm{Ti}$. All of these will be dealt with in the calculations. $\mathrm{PtTi}_{3}$ forms in the Frank-Kasper type $\mathrm{Cr}_{3} \mathrm{Si}$ (A15) phase which is "ill-packed" in the sense that it involves linear chains of $T i$ atoms which are compressed to abnormally sinall nearest-neighbor approaches along the chain lines. Calculations will be reported for $P t T i_{3}$ in the $\mathrm{Cr}_{3} \mathrm{Si}$ and, for comparison, in the $\mathrm{Cu}_{3} \mathrm{Au}$ (which is not observed) structures, each having the observed $\mathrm{Cr}_{3} \mathrm{Si}$ molecular volume. PtTi forms in the $\mathrm{AuCd}$ structure at low temperatures and undergoes a Martensitic transformation to the high temperature $\mathrm{CsCl}$ structure at $\sim 1100^{\circ} \mathrm{C}$. This is a higher temperature than is usually encountered for such transformations. The $C s C l$ phase would appear to be the one involved in the $\Delta H$ measurements but both structures (as well as the cubic $C u A u I$ structure) will be represented in the calculations using th - low temperature unit cell volume characteristic of the $\mathrm{AuCd}$ phase. Three different structures have been reported for $P t_{3} T i$ at or near 3:1 composition. The most commonly encountered is the $C u_{3} A u$ and it is the one reached [90 Sel] in the calorametric measurement. The other two structures involve 16 and 28 atoms in 
their unit cells, making them costly for total energy estimates and so the $\mathrm{Pt}_{3} \mathrm{Ti}$ calculations will be limited to the $C u_{3} A u$ structure. These is also reported to be a $P t_{5} T_{i_{3}}$ with 16 atoms per unit cell for which there is no experimental $\Delta H$. This too will be neglected. $\mathrm{Pt}_{8} \mathrm{Ti}_{i}$ is an ordered structure with the atoms on a slightly distorted $f c c$ lattice, the distortions in $P t$ positions arising from accomodation to the presence of larger $T i$ atoms. The calculations have been done with the full potential LASTO scheme with valence electron spin orbit coupling when obtaining both the total energies of the compounds and the reference total energies of $T i$ and $P t$.

The $\Delta H$ results are plotted in Fig. 4. The top panel is a rough tracing of the phase diagram [91 Mof] of the Pt-Ti system. The hatched areas indicate the compounds of concern here, all of which occur over a finite composition range. The dashed line at $\sim 1100^{\circ} \mathrm{C}$ indicates the transition from the low to the high temperature PtTi phase. The bottom panel compares the calculations with the experimental data. The agreement of the calculated $\Delta H$ with experiment is of the order of the scatter in the experimental values seen for those cases for which more then one value has been reported. This agreement is one tenth of an $\mathrm{eV}$ per atom or better (we expect that some of the more recent experimental data has been determined to an accuracy which is better than this).

The two phases which are not observed experimentally, the open circles which correspond to $\mathrm{PtTi}(\mathrm{AuCuI})$ and $\mathrm{PtTi}_{3}\left(\mathrm{Cu}_{3} \mathrm{Au}\right)$ are seen to have $\Delta H$ which are less bound than those appropriate to the observed low temperature phases at those compositions. Going from one composition to another, the calculated $\Delta H$ indicate the coexistence of the phases which do occur. Consider the dashed lie drawn between the $\Delta H$ for the $A u C d$ structure and the zero for pure $T i$. The fact that the calculated $\Delta H$ for $\operatorname{PtTi}_{3}\left(\mathrm{Cr}_{3} \mathrm{Si}_{i}\right)$ lies above this line indicates that $\mathrm{Pt}_{3} \mathrm{Ti}$ is calculated to be stable relative to a two phase mix of PtTi $(A u C d)$ and pure $\cdots i$ at the 1:3 composition. The fact that the PtTi $(A u C d)$ point lies above a line drawn between the $\mathrm{Pt}_{3} \mathrm{Ti}\left(\mathrm{Cu}_{3} \mathrm{Au}\right)$ and $\mathrm{PtTi}_{3}\left(\mathrm{Cr}_{3} \mathrm{Si}\right) \Delta \mathrm{H}$ indicates it to be stable relative to a two phase mix of $P t_{3} T i$ and $P t T i_{3}$ at $1: 1$ composition and 
similar arguments may be extended to show that $P t_{3} T i$ and $P t_{8} T i$ are also stable relative to competing mixes of adjacent phases.

The use of full potentials is important to the present results. The total energy of a solid goes down upon going from a calculation based on a muffin-tin potential (spherical within an atomic sphere and constant in the interstitial region) to one based on a full potential. Whether the structural energy of Eq. (1) or the heat of formation of Eq. (2) increases or decreases, depends upon which total energies in these expressions increase the most. In the case of an ill-packed compound such as $\mathrm{PtTi}_{3}\left(\mathrm{Cr}_{3} \mathrm{Si}\right)$ compared to the well-packed elemental $P t$ and $T i$ systems, one expects the set of full potential energies to yield the larger $\Delta H$. This is the case, the increase being $0.1 \mathrm{eV} /$ atom. The best packed of the compounds, in the sense of having the highest atomic site symmetries, is $\mathrm{PtTi}(\mathrm{CsCl})$ and its $\Delta H$ loses $0.15 \mathrm{eV} /$ atom upon going to the full potential. Here the full potential treatment is more important to the energies of elemental Pt and Ti than it is to that of the compound! This is also the case for the other compounds, though the shifts in their $\Delta H$ are much less. Going from muffin-tin to full potentials thus causes the $\Delta H$ of one compound, as compared with another, to vary typically by $0.1-0.15 \mathrm{eV} /$ atom with a shift of $0.25 \mathrm{eV} /$ atom when comparing $\mathrm{PtTi}(\mathrm{CsCl})$ to $\mathrm{PtTi}_{3}\left(\mathrm{Cr}_{3} \mathrm{Si}\right)$. This is substantial.

In Fig. 4 we see that the low temperature PtTi $(A u C d)$ phase is calculated to be stabler than the high temperature $P t T i(C s C l)$ as it should be, and that the calculated $C s C l$ structure's $\Delta H$ is in reasonable accord with experiment. The $C u A u I$ structure, which does not occur, is found closer in energy to the $\mathrm{AuCd}$ than is the CsCl. The difference in calculated $\Delta H$ of the $A u C d$ and $C s C l$ phases, $0.16 \mathrm{eV} /$ atom, seems rather large. However, at the phase transition, the temperature times the entropy change, $\Delta S$, must equal $\Delta H$ and the transition temperature here is quite high. Presumably vibrational entropy is the primary contribution to $\Delta S$, but we are not prepared to estimate this here. There are also electronic contributions [ $75 \mathrm{Gri} ; 84 \mathrm{Wat}$ ] and given the densities of states at the Fermi level for the two phases, these may be estimated. Using the present band theory 
calculations, an electronic $\Delta S$ of the right sign to account for $\sim 20 \%$ of the calculated $\Delta H$ may be obtained. It remains to be seen if the vibrational entropy can account for the remainder. There is some experimental basis for taking an $\Delta H$ of this magnitude seriously for such systems. $H f N i$ is reported [ $83 \mathrm{Nas} ; 91 \mathrm{Mof}$ ] to undergo an allotropic transformation at $1170^{\circ} \mathrm{C}$. (Note: $H f$ and $N i$ lie in the same columns of the Periodic Table as $T i$ and $P t$ respectively.) Gachon has pointed out $[91 \mathrm{Gac}]$ that the experimentalists responsible for the PtTi data of Figure 4 have also cbtained $\Delta H$ for $H f N i$, but at room temperature in one case and above the phase transition in another. Some uncertainty must be applied to the difference in the two heats but the low temperature heat is $\sim 0.11 \mathrm{eV} /$ atom larger in magnitude. This is consistent in sign and magnitude with the present calculations for PtTi though it should be noted $H f N i$ would appear to involve a transformation between a different pair of structural phases.

\section{Discussion}

In this paper we have concentrated on the results of total energy calculations which are accurate estimates of what is yielded by band theory employing the Hedin-Lundqvist class of local potentia!. For the Pt-Ti system, the heats of formation calculated are in accord with the known phase diagram and with the experimental heats obtained for this system. Differences in the calculated heats between the more rigorous full potential treatment and the computationally much more economic muffin-tin potential can be substantial when measured on a scale of what one requires for phase diagram constructs. We expect that these changes are primarily associated with the treatment of the charge density (and in turn the potential) in the interstitial region and, in particular, in the variation in that charge density. This, in turn, is coupled to the choice of the atomic sphere radii used in the calculations (a common pair of radii were used for all the Pt-Ti compounds here). The full potential $\Delta H$ are insensitive to this choice, but the muffin-tin results are not. This issue requires further investigation if one wishes to rely on atomic sphere or muffin-tin calculations of total energies. 
In the case of total energy estimates relevent to multilayer and adlayer behavior we encountered a factor not entering standard phase diagram considerations, namely the importance of surface energies to the energetics of fabricating such systems. Given the surface energies as well as the other terms, much of what has been observed experimentally can be rationalized quantitatively.

In the case of the structural promotion energies of the elemental transition metals, we have revisited a long standing matter of contention between band theory and CALPHAD practitioners. Comparison with the estimates of Saunders et al. [88 Sau], who pushed the $f c c-b c c$ energy differences as close as possible to band theory estimates while remaining consistent with CALPHAD constructs, shows the two to be in semiquantitative agreement across most of the transition metal rows. Problems remain with the $f c c-h c p$ energy differences which are smaller in magnitude than the $f c c-b c c$ ones and which may be embroiling us with the eternal problem of successfully dealing with the sign of an essentially zero-valued quantity. The problem centers on the $f c c-h c p$ energy differences for the bcc metals of the $V$ and $C r$ columns of the periodic table where CALPHAD concerns require the $h c p$ to be the stabler of the two structures so as to deal with the ocurrence of $h c p$ phases of intermediate alloy composition. Band theory has consistently indicated the $f c c$ to be stabler. This disagreement may simply be a failure of band theory employing the class of potential employed here. However, from our biased viewpoint we think the disagreement represents a shortcoming in conventional phase diagram constructs involving not having listened sufficiently to Hume-Rothery. Hume-Rothery type arguments would argue that the dominent factor controling the appearance of intermediate $h c p$ phases would be the average valence electron to atom ratio of these systems. Has this been sufficiently accounted for in the constructs?

Estimates of total energies (and of total energy differences) vary by many orders of magnitude in computational effort. These range from model Hamiltonian estimates, such as those of Miedema's, which can, if need be, be done by hand to calculations such 
as those which we have described here. Clearly, taking the ratio of quality of result to computational effort, schemes such as Miedema's represent "best buys." In addition to structural questions, total energy calculations can be used to obtain parameters for simpler model Hamiltonians, spectroscopic information (via $\triangle S C F$ calculations), and information concerning phonons (using the so-called "frozen phonon" method), as well as providing an important ingredient in first-princip'es molecular dynamics methods. Although some readers may disagree in the case of the structural energies discussed above, it would appear that the "all bells and whistles" estimates of heats of formation and of structural energy differences represented here are in reasonable accord with experiment and may reasonably be employed in thermodynamic constructs of phase diagrams. Despite rapid increases in computer capability, it will likely be the schemes of intermediate computational complexity which will be primarily relied on to obtain estimates for complicated alloy systems. These can provide physical insights into bonding trends, can allow scans over many competing systems (as in Zunger's paper in this volume), and can provide quantitatively meaningful estimates of heats providing sufficient care is taken when making such estimates. 


\section{Acknowledgment}

This work was supported under contract DE-AC02-76CH00016 with the Division of

Materials Sciences, U.S. Department of Energy and by a grant of computer time at the National Energy Research Supercomputer Center (NERSC), Livermore, California. 


\section{References}

[67 Rud] Phase Stability in Metals and Alloys, edited by P. S. Rudman, J. Stringer, and R. I. Jaffe (McGraw-Hill, New York, 1967).

[70 Kau] L. Kaufman and H. Bernstein, Computer Calculations of Phase Diagrams (Academic, New York, 1970).

[71 Hed] L. Hedin and B. I. Lundquist, J. Phys. C4, 2064 (1971).

[75 Gri] G. Grimvall, Phys. Sci. 12, 173 (1975).

[77 Pet] D. Pettifor, CALPHAD $\underline{1}, 305$ (1977).

[83 Nas] P. Nash and A. Nash, Bull Alloy Phase Diag. $\underline{4}, 250$ (1983).

[84 Wat] R. E. Watson and M. Weinert, Phys. Rev. B30, 1641 (1984).

[85 Col] C. Colinet, A. Pasturel, and P. Hicter, CALPHAD $\underline{9}, 71$ (1985).

[85 Dav] J. W. Davenport, R. E. Watson and M. Weinert, Phys. Rev. B32, 4883 (1985).

[85 Skr] H. Skriver, Phys. Rev. B31, 1909 (1985).

[88 Gui] A. F. Guillermet and M. Hillert, CALPHAD $\underline{12}, 237$ (1988).

[88 Sau] N. Saunders, A. P. Miodownik, and A. T. Dinsdale, CALPHAD $\underline{12}, 351$ (1988).

[88 Top] L. Topor and O. J. Kleppa, Metall. Trans. 19A, 1827 (1988).

[89 Jia] L. Q. Jiang, M. W. Ruckman, and M. Strongin, J. Vac. Sci. Technol. A7, 2016 (1989).

[89 Wei] M. Weinert, R. E. Watson, J. W. Davenport, and G. W. Fernando, Phys. Rev. $\underline{\mathrm{B} 39}, 12585$ (1989). 


\section{References}

Cont.

[90 Fer] G. W. Fernando, R. E. Watson, M. Weinert, Y. J. Wang, and J. W. Davenport, Phys. Rev. B41, 11813 (1990).

[90a Sel] N. Selhaoui, Etude Thermo Dynamique de Composes Binaires de Metaux de Transition par Calorimetric Hautes Temperatures. Modelisation Numerique de Diagrammes de Phases. Confrontation Experiences-Modeles, (Ph.D Thesis, University of Nancy, France, 1990).

[90b Sel] N. Selhaoui and J.-C. Gachon, Anales de Fisica B86, 57 (1990).

[91 Gac] J.-C. Gachon, (private communication).

[91 Mof] W. G. Mofatt, The Handbook of Binary Phase Diagrams (Genium, Schenectady, New York, 1991).

[91 Str] M. Strongin and A. Gibaud, (private communication).

[92 Fer] G. W. Fernando, R. E. Watson, and M. Weinert, Phys. Rev. B (submitted). 


\section{Figure Captions}

Fig. 1 The $f c c \rightarrow b c c$ structural energy differences for the elemental $4 d$ metals. The black dots were obtained from relativistic LASTO calculations employing local density potentials. The open triangles are the estimates of Saunders et al. [ $88 \mathrm{Sau}$ ] of what is thermodynamically consistent with alloy phase behavior. These are of the same sign but measurably larger in magnitude than Kaufman's estimates [70 Kau].

Fig. 2 The variation in total energy as a function of lattice constant $a$ (plotted as a ratio of $a$ with respect to the observed lattice constant $\left.a_{o b s}\right)$ for $f c c R h, P d$, and $A g$, and for $h c p R u$ (for which the $c / a$ ratio was kept at its observed value). The zero of each plot corresponds to the lowest total energy of a set of plotted points which is indicated by an open circle.

Fig. 3 The total energies measured relative to the calculated ground state energies of $R u$, $R h, P d$, and $A g$ where these metals have been constrained to have the (110) planar lattices appropriate to $b c c M o$ and $N b$. The energies are plotted as a function of the distance between (110) planes, $c_{M o}$ and $c_{N b}$ corresponding to the observed interplanar spacing appropriate to $b c c M o$ and $N b$ respectively. Open circles indicate the lowest calculated energies on any given curve and the vertical arrows indicate the planar spacing such that the lattice has the same atomic volume as was obtained in Fig. 2 for $R u, R h, P d$, or $A g$. The horizontal lines in the middle of the Figure are the calculated $f c c \rightarrow b c c$ (for $R u h c p \rightarrow b c c$ ) structural energy differences.

Fig. 4 The upper section shows a rough tracing of the phase diagram of the $P t-T i$ alloy system $[91 \mathrm{Mof}]$. The hatching indicates the ordered phases of concern to the calculations represented in the lower panel which displays experimental and calculated heats of formation for varying Pt-Ti composition. The crosses indicate calorametric measurements and the $x$ 's $e m f$ measurements. The calculated values rely on full potential, fully relativistic LASTO calculations. The filled circles correspond to $P t_{8} T i$, 
$\mathrm{Pt}_{3} \mathrm{Ti}\left(\mathrm{Cu}_{3} \mathrm{Au}\right), \mathrm{PtTi}_{3}\left(\mathrm{Cr}_{3} \mathrm{Si}\right)$ and the high temperature $\mathrm{PtTi}(\mathrm{Cs} C l)$ phase. The filled square corresponds to the low temperature PtTi $(A u C d)$ phase and the open circles to the PtTi $(C u A u I)$ and $P t T i_{3}\left(C u_{3} A u\right)$ structures which are not reported to occur. Note that for the 50/50 composition, it is the high temperature PtTi $(\mathrm{CsCl})$ structure whose calculated $\Delta H$ should be compared with experiment. 


\section{Table I}

Calculated Surface Energies $\gamma$ [89 Wei]

\begin{tabular}{|c|c|c|}
\hline Surface & $\gamma($ eV/atom $)$ & $\gamma\left(J / \mathrm{m}^{2}\right)$ \\
\hline $\mathrm{Nb}(110)$ & 1.4 & 2.9 \\
$(010)$ & 2.1 & 3.1 \\
\hline $\operatorname{Ag}(001)$ & 0.7 & 1.3 \\
$(110)$ & 1.0 & 1.4 \\
\hline $\operatorname{Pd}(001)$ & 1.1 & 2.3 \\
$(110)$ & 1.7 & 2.5 \\
\hline
\end{tabular}




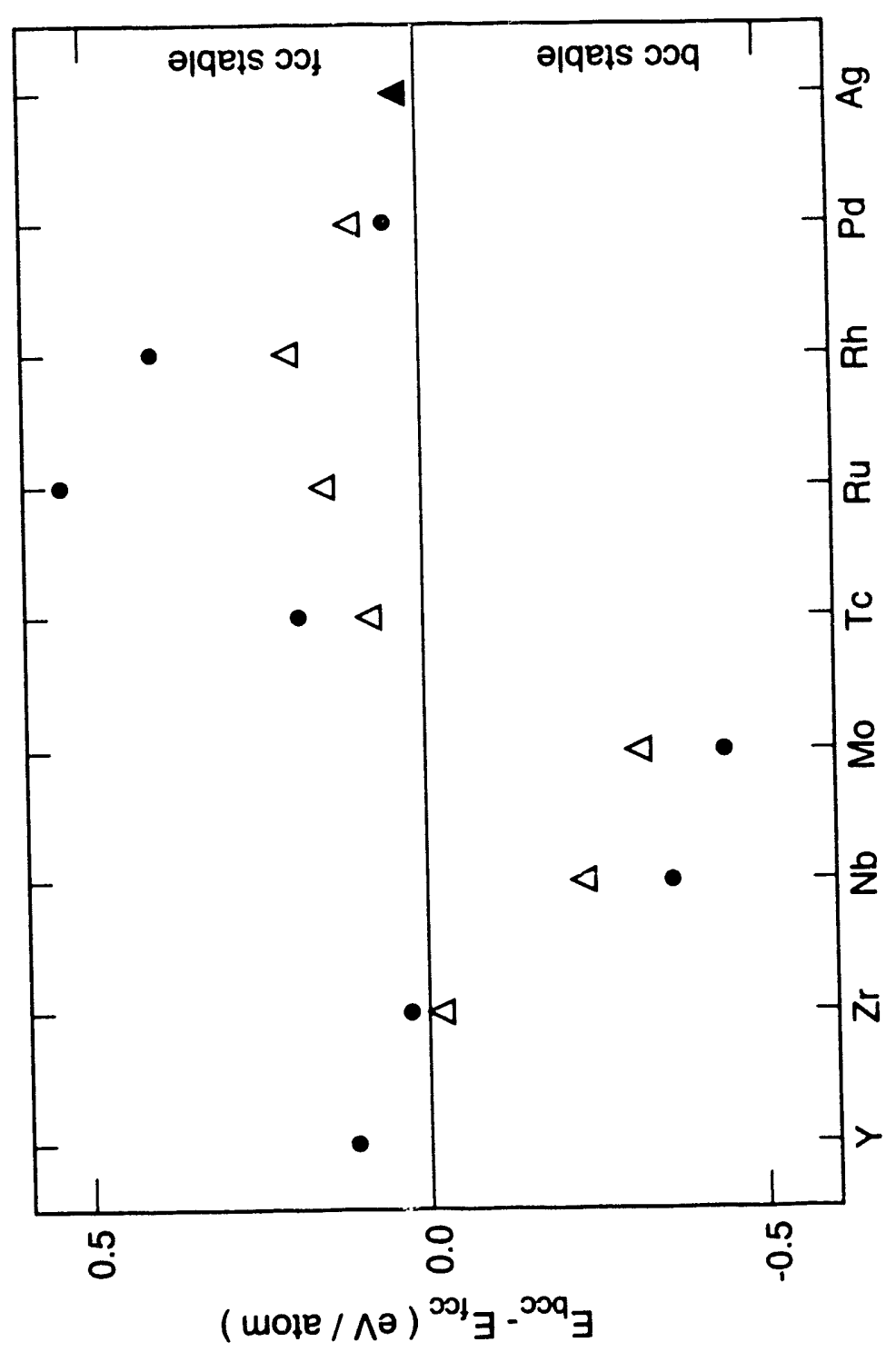

$\overline{1}$ 


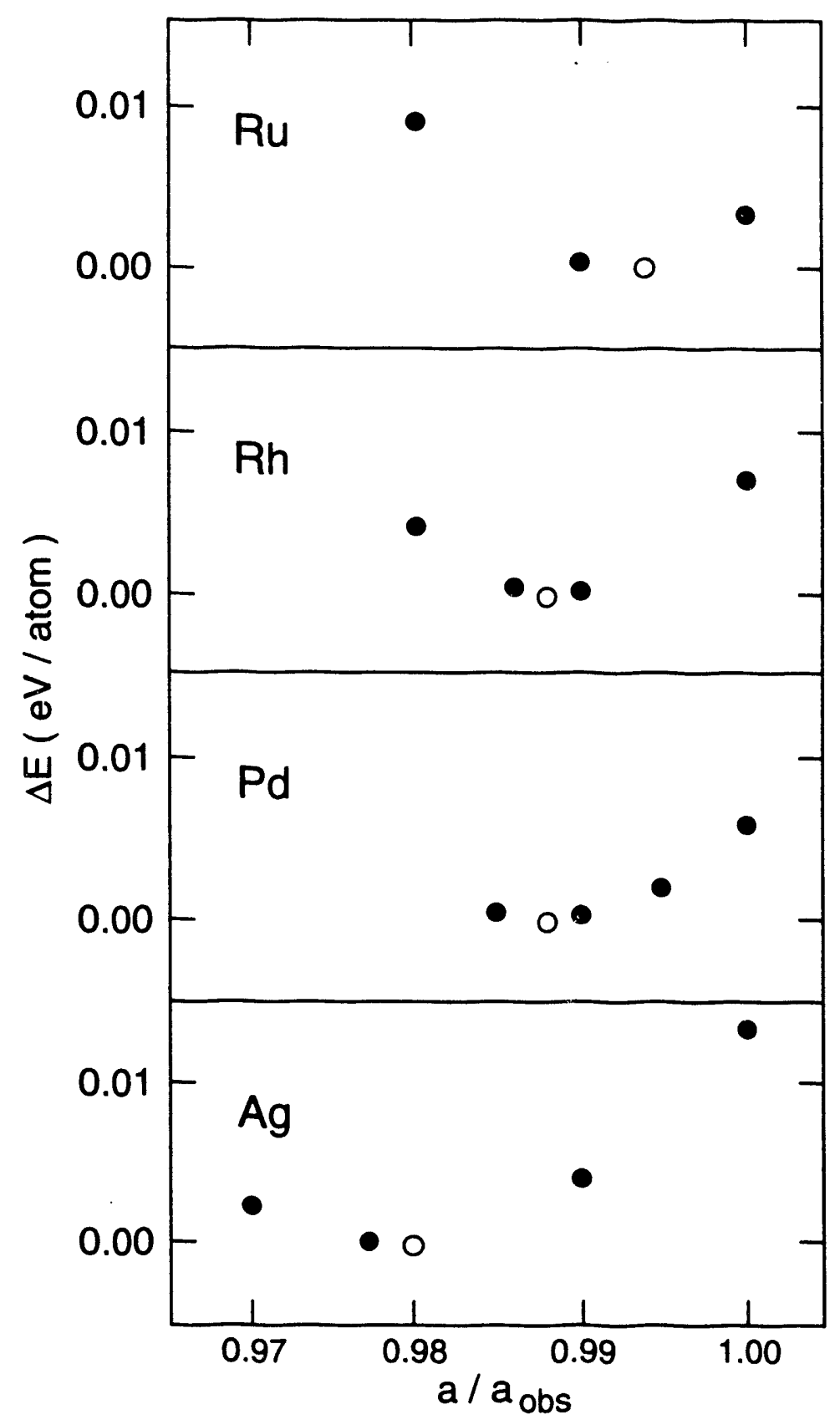

$=\lg 2$ 


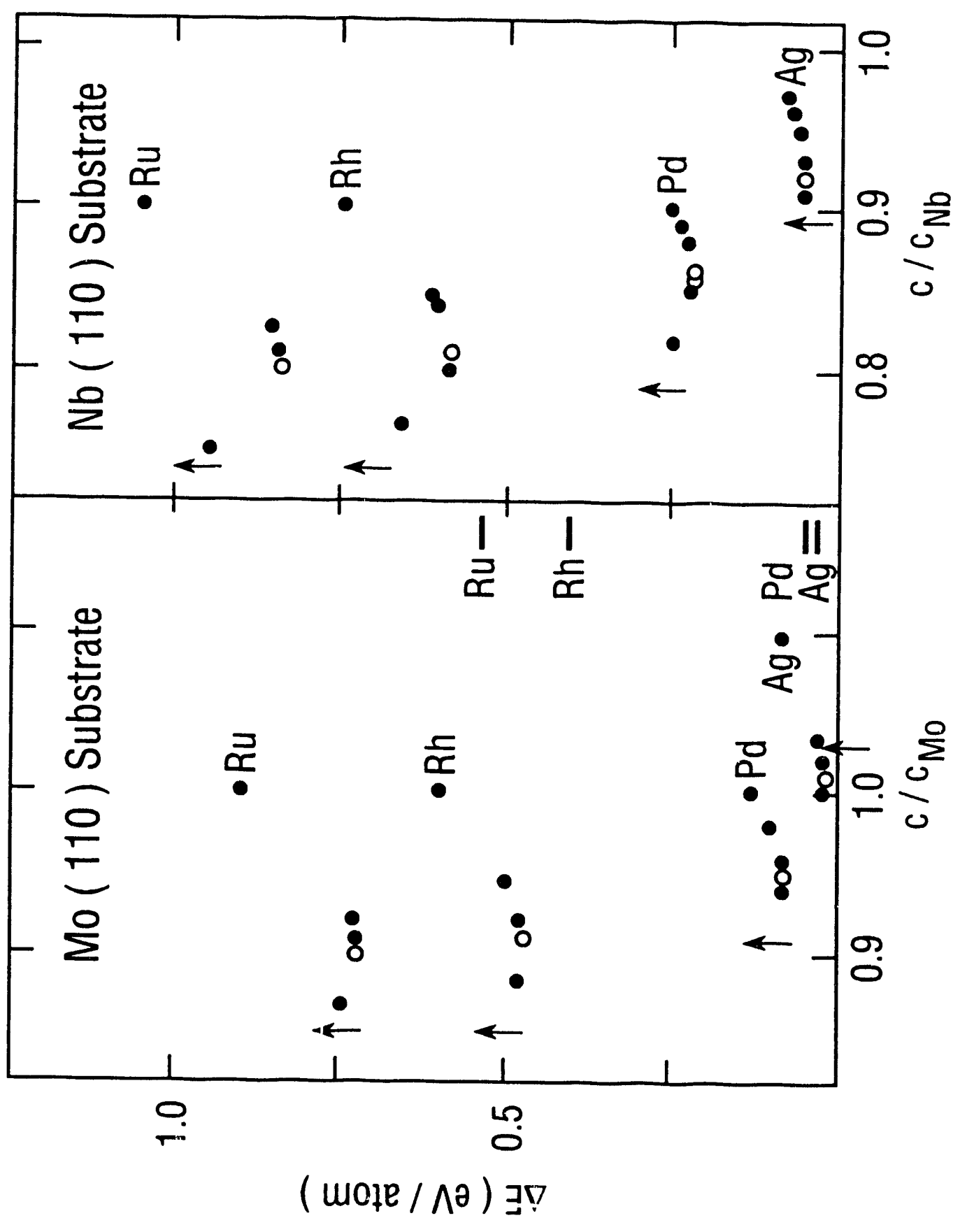

내 ถึ 


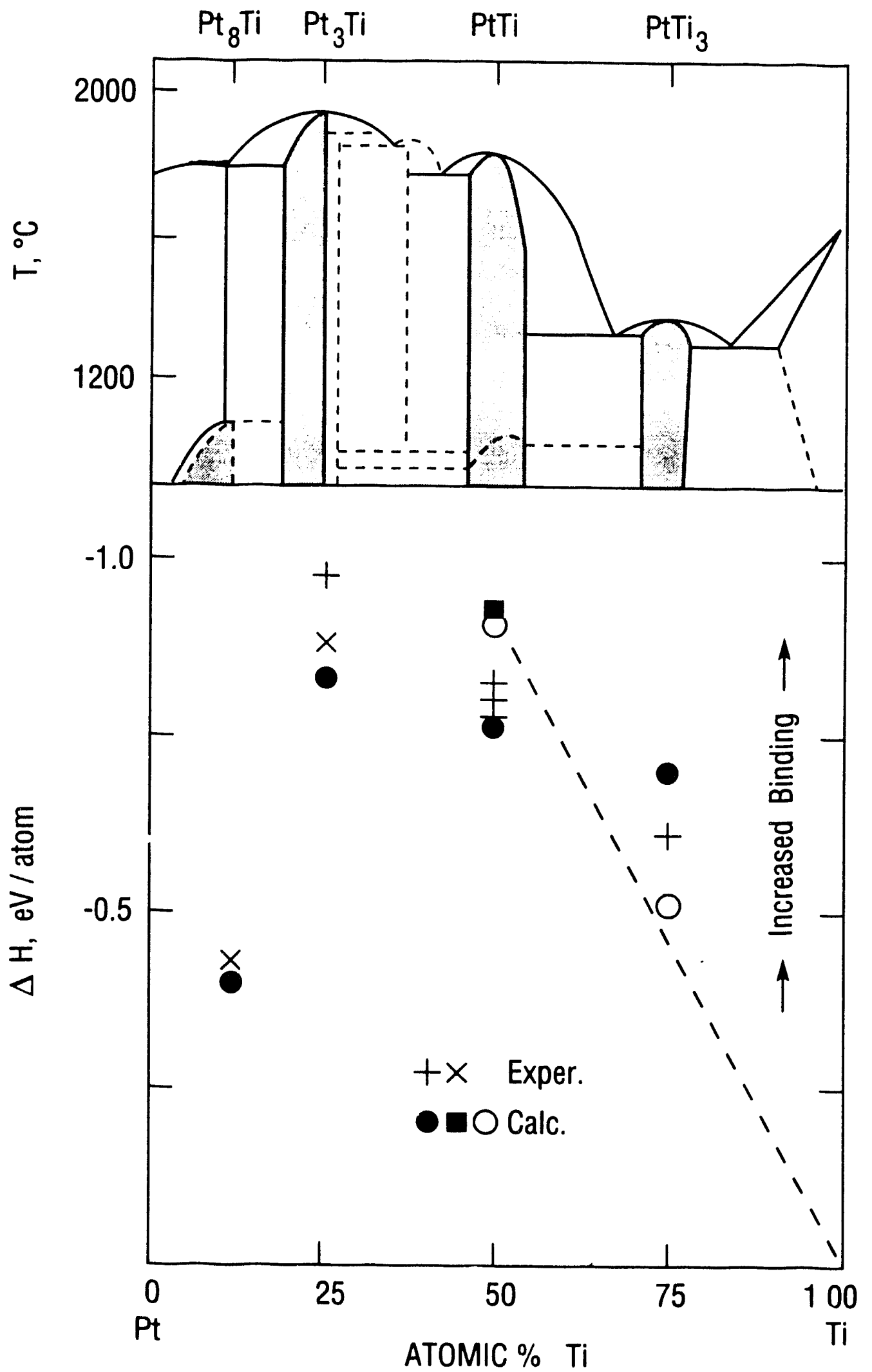

Figy

WAT 3-1 11/90 IR 

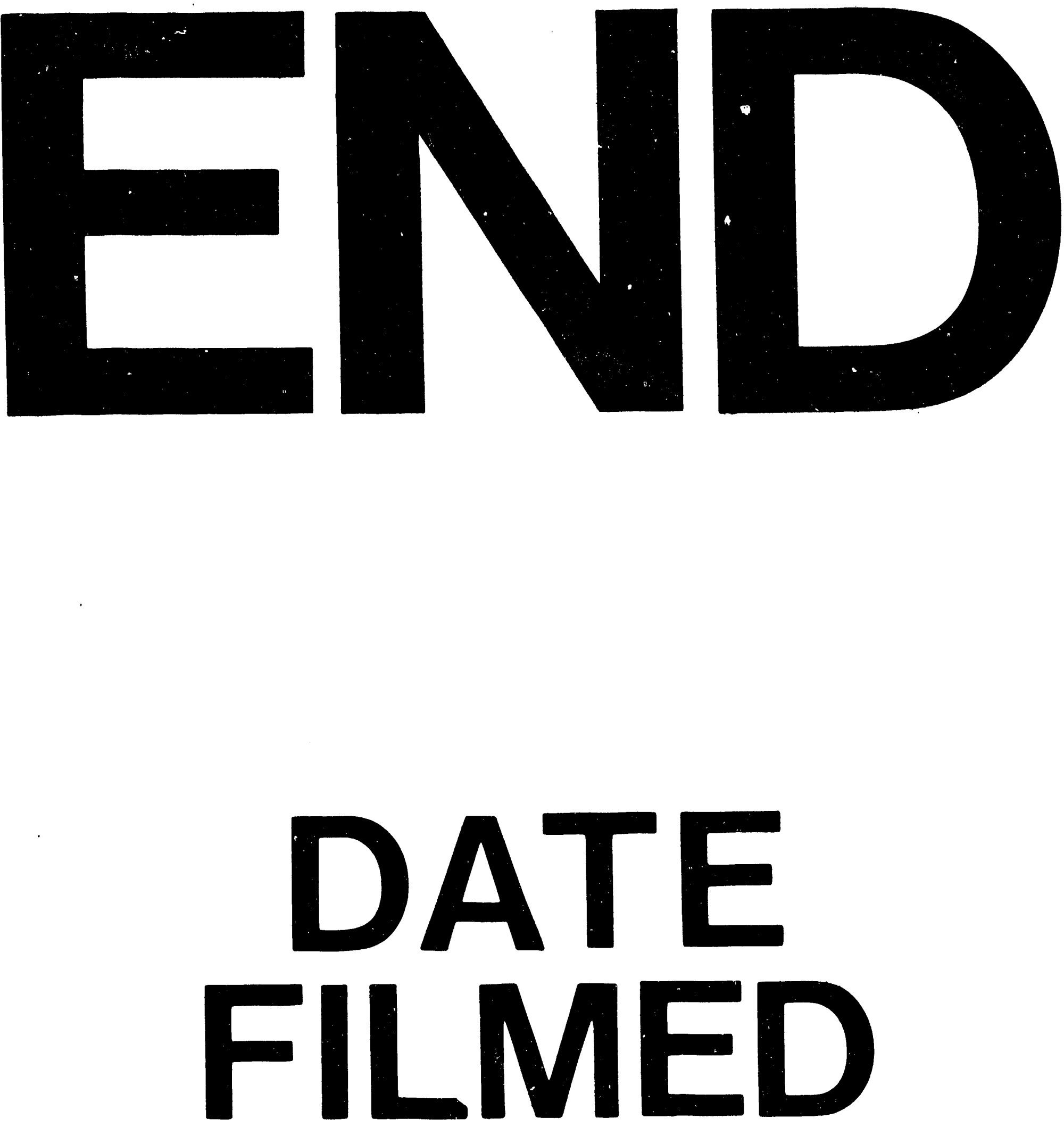

i

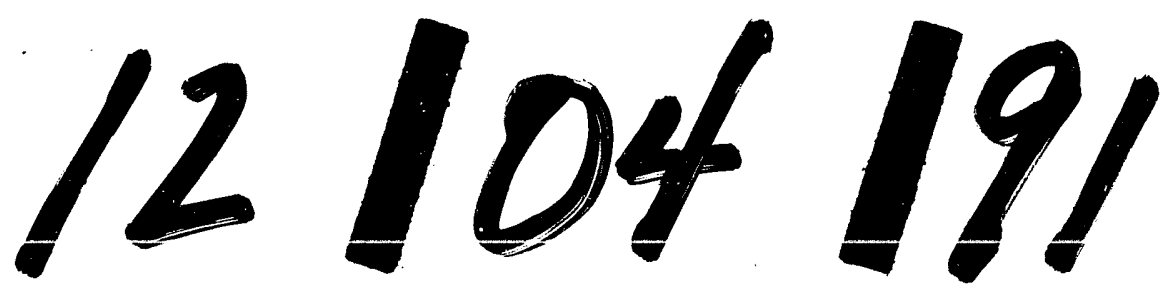

